\title{
Pheromonal influences on sociosexual behavior in young women
}

\author{
Norma L. McCoy*, Lisa Pitino \\ Department of Psychology, San Francisco State University, San Francisco, CA 94132-4168, USA
}

Received 27 August 2001; received in revised form 19 November 2001; accepted 21 November 2001

\begin{abstract}
A double-blind, placebo-controlled study of a synthesized putative female pheromone was conducted with regularly menstruating, university women $(N=36$, mean age $=27.8)$. The pheromone formula was derived from earlier work investigating the underarm secretions of fertile, sexually active, heterosexual women. A vial of either synthesized pheromone or placebo was selected blindly and added to a subject's perfume. Subjects recorded seven sociosexual behaviors and reported them weekly across three menstrual cycles. Beginning with Day 8 of each cycle, the first cycle contained a 2-week baseline period followed by an experimental period of as many as 3 weeks each from the next two cycles for a maximum of 6 weeks. The 19 pheromone and 17 placebo subjects did not differ significantly in age, weight, body mass index, dating status or ethnicity nor in reported accuracy, back-filling data, perception of a positive effect or perfume use. Placebo subjects were significantly taller than pheromone subjects. Except for male approaches, subjects did not differ significantly at baseline in average weekly sociosexual behaviors. A significantly greater proportion of pheromone users compared with placebo users increased over baseline in frequency of sexual intercourse, sleeping next to a partner, formal dates and petting/affection/kissing but not in frequency of male approaches, informal dates or masturbation. Three or more sociosexual behaviors increased over baseline for $74 \%$ of pheromone users compared with $23 \%$ of placebo users. We conclude that this synthesized pheromone formula acted as a sex attractant pheromone and increased the sexual attractiveness of women to men. (c) 2002 Elsevier Science Inc. All rights reserved.
\end{abstract}

Keywords: Female pheromones; Human sexuality; Sexual behavior; Sexual attraction; Women's sexuality

\section{Introduction}

The introduction of the term 'pheromone' to the scientific community in 1959 was followed by numerous studies involving animals as subjects. A pheromone was defined as

a substance excreted by an animal, to the outside of that individual, which is then received by another individual, classically of the same species, which then elicits some behavioral or developmental response related to the survival of the species [12].

If the chemical product of a body acts on another body some distance from the originating organism and serves the reproductive life of the species, that chemical is termed a pheromone. More recently, after reviewing the literature of human sex attractant pheromones, Cutler [4] has concluded that four behavioral effects or classes of pheromonal action

* Corresponding author. 4252 Newberry Court, Palo Alto, CA 943064138, USA. Tel.: +1-650-493-1457; fax: +1-650-494-8220.

E-mail address: mccoy@sfsu.edu (N.L. McCoy). have been experimentally demonstrated both in animals and humans. These classes of pheromonal action are the opposite-sex attractants, the same-sex repellants (territorial markers), mother-infant bonding attractants and those modulating the timing of the fertile cycle.

Strong evidence presented recently argues that in small mammals there are two different neural pathway systems mediating disparate pheromonal-type behavioral effects: the vomeronasal organ (VNO) and the olfactory epithelium [2]. The VNO is probably not involved in sexual attraction in humans or other mammals but could be involved in disruptive and/or timing signals such as altering the timing of a menstrual cycle or serving as a repellant, i.e. a territorial marker [3]. The potential role of the VNO in human pheromonal involvement remains controversial $[1,10,11,13,14]$.

While a growing philosophic commentary about human pheromones exists, the published literature offers few double-blind, placebo-controlled studies of human pheromonal effects. The first such investigations were a series by Cutler et al. [8] and Preti et al. [15]. These studies used pooled and frozen underarm secretions collected from apparently fertile, 
sexually active, young adults. Thawed after 1 year, filtered extracts from these secretions were used in double-blind, placebo-controlled research in which subjects applied them three times a week to the skin area above the lip and under the nose. Such use produced significant effects on the timing of women's menstrual cycles. In addition, a post hoc analysis revealed that significantly more women using the active substance compared with placebo engaged in weekly sexual intercourse $[5,6]$.

A subsequent study also showed that underarm secretions altered the timing of menstrual events [17]. These authors reported that daily application of extracts from preovulatory phase underarm secretions advanced the timing of ovulation by 1.7 days, while extracts from postovulatory underarm secretions delayed the onset of ovulation by 1.4 days. A double-blind, placebo-controlled study with male subjects [7] investigated a synthetic version of a pheromone naturally secreted by men for its effects on six sociosexual behaviors. Following a 2-week baseline period, a technician added either the substance or a placebo to the men's aftershave perfume. Significantly more men who used the aftershave with pheromone compared with those who used it with placebo increased over baseline in their frequency of sexual intercourse and sleeping next to a romantic partner. Increases over baseline for petting/affection/kissing as well as informal dating for pheromone compared with placebo users reached borderline levels of significance.

The present study investigates the effects of a synthesized pheromone formula on the sexual attractiveness of women. This study was designed as a double-blind, placebo-controlled investigation following a protocol requiring prospective daily recording of sociosexual behaviors over three consecutive menstrual cycles. The major hypothesis was that a greater proportion of subjects using pheromone than those using placebo would show increases over baseline in intimate sociosexual behavior involving a male partner.

\section{Method and materials}

\subsection{Subject recruitment}

Subjects were recruited using announcements in classes at San Francisco State University. Women were asked to participate in an experiment described as testing whether a female pheromone added to their perfume would "increase the romance in their lives." Criteria for inclusion were that subjects be heterosexual, regularly menstruating, neither married nor cohabiting with a man, in self-reported good health and not currently using an oral contraceptive. Subjects were screened for adequate social skills based upon the revised Eysenck Personality Inventory (EPQ-R) [9]. A score more than 2 standard deviations above the Eysenck's manual standardized neuroticism, extraversion and psychoticism statistics was criteria for disqualification. As an inducement to complete the study, subjects were informed that their treatment condition would be revealed at the end of the study, and at that time, all placebo subjects would receive a vial of the synthesized pheromone formula.

\subsection{Sample}

Of 47 respondents who completed an intake form, 36 women completed the study. Nineteen women received a pheromone vial in the experimental period, while 17 women received placebo. Four of 11 dropouts did not complete the baseline period. The remaining seven completed the baseline period and received a coded vial. Six subjects (four pheromone and two placebo) quit before the end of the first experimental cycle and one placebo subject did not return data prospectively as agreed. Of the 36 who completed the study, one placebo subject provided behavioral data for only one experimental cycle, while 35 provided data for two experimental cycles. A review of the characteristics of those who completed the study revealed no systematic bias in the makeup of the pheromone compared with the placebo group (see Table 1). No subject reported using oral contraceptives during the study.

Subjects ranged in age from 19 to 48 years with an overall mean age of 27.8 years $($ S.D. $=6.7)$. Average age of subjects in the pheromone group was not significantly different from that of the placebo group but a trend is noted $[t(24.8)=-1.74, P=.094$ (S.E. $=2.25)]$. Although subjects selected their vial blindly, subjects in the placebo group were significantly taller than subjects in the pheromone group $[t(33)=-2.05, P<.05$ (S.E. $=0.85)]$ while the two groups did not differ significantly for weight $[t(34)<1]$. Body mass index (weight/height ${ }^{2}$ ) was not significantly different for pheromone and placebo groups $[t(33)<1]$ nor was the length of the menstrual cycle at baseline $[t(34)<1]$.

Ethnicity in each group revealed no appreciable differences. For the pheromone group, 9 of 19 were White, 2 were Filipino and 1 each listed herself as Latina-Peruvian, Portuguese, Latina-Nicaraguan, Chinese, Mixed, Brazilian, White-Native American and Chinese American. Among the placebo group, 10 of 17 were White and 1 each listed herself

Table 1

Initial age, height, weight, body mass index, menstrual cycle length and relationship status for subjects by treatment group

\begin{tabular}{|c|c|c|c|c|}
\hline & \multicolumn{2}{|c|}{ Pheromone $(n=19)$} & \multicolumn{2}{|c|}{ Placebo $(n=17)$} \\
\hline & $M$ & S.D. & $M$ & S.D. \\
\hline Age (years) & 25.6 & 4.6 & 29.5 & 8.2 \\
\hline Height (in.) & 63.7 & 2.7 & $65.4^{\mathrm{a}}$ & 2.2 \\
\hline Weight (lb) & 134.3 & 25.1 & 130.9 & 21.7 \\
\hline Body mass index & 23.3 & 4.4 & 23.3 & 2.7 \\
\hline Menstrual cycle length & 28.8 & 4.9 & 29.8 & 4.9 \\
\hline Relationship status & $n$ & $\%$ & $n$ & $\%$ \\
\hline Not dating & 8 & 42 & 9 & 53 \\
\hline Dating & 8 & 42 & 1 & 6 \\
\hline Dating steadily & 3 & 16 & 7 & 41 \\
\hline
\end{tabular}

${ }^{\text {a }} n=16$. 
Table 2

Definition of baseline and experimental weeks

\begin{tabular}{ll}
\hline Baseline & Week 1: Days 8-14 \\
& Week 2: Days 15-21 \\
Experiment C1 (cycle 1) & Week 1: Days 8-14 \\
& Week 2: Days 15-21 \\
& Week 3: Days 22-28 \\
Experiment C2 (cycle 2) & Week 1: Days 8-14 \\
& Week 2: Days 15-21 \\
& Week 3: Days 22-28 \\
\hline
\end{tabular}

as American Indian, Indian Caucasian, Mexican American, Japanese, American Indian-Jew, Hispanic and Assyrian.

\subsection{Procedure}

At the first meeting, subjects signed an informed consent form approved by the Human Subjects Committee at San Francisco State University explaining the study protocol. They then filled out a personal information sheet asking about their sexual, reproductive and contraceptive history and completed the Eysenck Personality Inventory. The researcher provided calendars for the baseline period, and subjects selected a code name and recorded it on the top of each calendar. The researcher explained the definitions of the seven sociosexual behaviors and showed the subject how to record the occurrence of menses and behavior. The seven sociosexual behaviors were petting/ affection/kissing, sleeping next to a romantic partner, sexual intercourse, formal dates (preagreed and scheduled in advance), informal dates (not prescheduled), male approaches and masturbation.

At this first meeting, subjects also brought at least $55 \mathrm{ml}$ of her preferred perfume. If the perfume was in a spray bottle, subjects were provided with a clean $60-\mathrm{ml}$ cobalt blue glass bottle with a screw top. Application of the perfume was carefully demonstrated - two or three dabs under the nose, cheeks, behind the ears at least every other day. The researcher and each subject exchanged telephone numbers and best times for communicating. Subjects were instructed to start recording data on the first day of their next menses, which marked the onset of the baseline period and to mail the calendars at the end of each week. Finally, subjects agreed to telephone the researcher on the first day of menses in order to set an appointment for Day 22 of this first cycle and begin the experimental phase of the study. Between meetings, the researcher called subjects to followup missing calendars and to set-up or confirm an appointment for Day 22 of the first cycle.

At the second meeting, on about Day 22, the researcher checked the correct use of the perfume and the recording on the calendar, passed out remaining calendars and envelopes and reviewed the plan for the experimental phase of the study. Each subject selected a box from a tray of boxed vials that were identical in appearance. Once selected, code 'A' or ' $B$ ' was revealed in two places, both on the bottom of the box and on the label of the 5-ml glass vial inside the box.
The researcher wrote the ' $\mathrm{A}$ ' or ' $\mathrm{B}$ ' code on each of the subjects' calendars. At that time, each subject wrote her code name on each of her calendars as well. The researcher added the complete contents of the vial to $55 \mathrm{ml}$ of the subject's perfume. Each subject agreed to continue wearing perfume, recording daily on her calendars and mailing them weekly as in the baseline period. The third interview was conducted by telephone. The researcher completed a debriefing form with the information provided by each subject. Subjects were asked to rate their accuracy in recording behavior, the proportion of time they back-filled data and their best guess as to whether they had used pheromone or placebo in their fragrance.

\subsection{Pheromones}

Underarm secretions from sterile pads worn by sexually active heterosexual, fertile women were investigated in previous research [16]. Based on knowledge from this research, Cutler, a coauthor of the earlier work, derived the formulation used here as a proprietary, synthesized human female pheromone formula under the trade name Athena Pheromone 10:13. This formula is commercially available and its chemical identity will be disclosed when the patent process is completed. The Athena Institute for Women's Wellness, Chester Springs, PA supplied the researchers with 23 coded vials of pheromone formula and 23 of placebo. After all data had been collected, the researchers were informed by Athena Institute that the ' $\mathrm{A}$ ' vial contained the pheromone formula in SD40 alcohol and the 'B' vial contained plain SD40 alcohol. Both additives were odorless except for a faint alcohol scent; when added to a fragrance, neither was recognized by the wearer as having changed the aroma of her fragrance.

\subsection{Sociosexual behavior}

During the 2-week baseline and the 6-week trial, each subject's total number of days per week for each of seven sociosexual behaviors was counted. Because some women specifically abstain while others specifically engage in sexual behavior during the time of menstrual bleeding, the first 7 days of each of the three menstrual cycles were

Table 3

Comparison of average weekly days of each of the seven sociosexual behaviors at baseline for the placebo and pheromone groups

\begin{tabular}{|c|c|c|c|}
\hline & Pheromone & Placebo & $t$ \\
\hline Petting/kissing/affection & 1.76 & 2.35 & $<1$ \\
\hline Sleeping next to a romantic partner & 0.97 & 2.26 & $1.87^{*}$ \\
\hline Sexual intercourse & 0.63 & 1.26 & 1.40 \\
\hline Formal dates & 1.14 & 0.68 & 1.00 \\
\hline Informal dates & 1.24 & 0.85 & $<1$ \\
\hline Male approaches & 2.45 & 1.40 & $2.24^{* *}$ \\
\hline Masturbation & 0.90 & 0.71 & $<1$ \\
\hline
\end{tabular}


Table 4

(a) Average weekly occurrence of four intimate sociosexual behaviors for pheromone and placebo users for the 2 baseline and 6 experimental weeks

\begin{tabular}{|c|c|c|c|c|c|c|c|c|}
\hline \multirow[b]{2}{*}{ Code name } & \multicolumn{2}{|c|}{$\begin{array}{l}\text { Petting/kissing/affection } \\
\text { (average days per week occurred) }\end{array}$} & \multicolumn{2}{|c|}{$\begin{array}{l}\text { Sleeping next to a romantic partner } \\
\text { (average days per week occurred) }\end{array}$} & \multicolumn{2}{|c|}{$\begin{array}{l}\text { Sexual intercourse (average } \\
\text { days per week occurred) } \\
\end{array}$} & \multicolumn{2}{|c|}{$\begin{array}{l}\text { Formal dates (average days } \\
\text { per week occurred) }\end{array}$} \\
\hline & Baseline & Experimental & Baseline & Experimental & Baseline & Experimental & Baseline & Experimental \\
\hline \multicolumn{9}{|l|}{ Pheromone } \\
\hline 1 Chase & 2.50 & 3.83 & 2.00 & 2.67 & 2.00 & 2.46 & 2.50 & 3.00 \\
\hline 2 Lolita & 1.50 & 2.40 & 1.00 & 2.40 & 0.00 & 0.60 & 1.50 & 1.00 \\
\hline 3 Barbie Doll & 5.50 & 3.78 & 4.00 & 4.48 & 1.50 & 2.02 & 1.50 & 2.52 \\
\hline 4 Narcisse & 0.00 & 0.67 & 0.00 & 0.00 & 0.00 & 0.17 & 0.00 & 0.00 \\
\hline 5 Coho & 0.00 & 0.00 & 0.00 & 0.17 & 0.00 & 0.00 & 0.00 & 0.00 \\
\hline 6 Ares & 3.50 & 2.67 & 2.50 & 1.67 & 2.00 & 1.50 & 1.50 & 1.67 \\
\hline 7 Gigi & 1.00 & 5.61 & 0.50 & 4.44 & 0.00 & 4.25 & 0.50 & 1.67 \\
\hline 8 Sarah Lee & 0.00 & 0.67 & 0.00 & 0.00 & 0.00 & 0.00 & 0.00 & 0.83 \\
\hline 941799 & 2.50 & 3.57 & 1.00 & 1.73 & 2.00 & 1.57 & 1.50 & 0.50 \\
\hline 10 Lynette & 1.00 & 1.00 & 0.00 & 0.33 & 0.00 & 0.00 & 0.50 & 1.83 \\
\hline 11 Potus & 3.00 & 2.33 & 3.00 & 0.33 & 0.00 & 0.00 & 2.00 & 2.00 \\
\hline 12 Randall & 0.50 & 0.60 & 0.00 & 0.40 & 0.00 & 0.40 & 0.00 & 0.40 \\
\hline 13 Saffron & 0.00 & 1.00 & 0.00 & 0.50 & 0.00 & 0.67 & 0.00 & 1.00 \\
\hline 14 Absinthe & 3.00 & 2.84 & 0.50 & 2.04 & 1.00 & 0.88 & 0.00 & 1.28 \\
\hline 15 Psychokity & 0.50 & 0.67 & 0.50 & 0.33 & 0.00 & 0.50 & 0.00 & 0.17 \\
\hline 16 Sammi & 3.50 & 3.80 & 2.00 & 1.60 & 1.50 & 1.20 & 4.00 & 2.20 \\
\hline 17 Yvette & 5.50 & 5.50 & 1.00 & 1.83 & 1.50 & 1.67 & 2.50 & 2.17 \\
\hline 18 Jeneve & 0.50 & 0.80 & 0.50 & 0.33 & 0.50 & 0.40 & 0.50 & 0.20 \\
\hline 19 Sunset & 0.00 & 0.00 & 0.00 & 0.00 & 0.00 & 0.00 & 1.00 & 0.40 \\
\hline \multicolumn{9}{|l|}{ Placebo } \\
\hline 1 Red & 1.50 & 2.60 & 1.50 & 2.40 & 1.50 & 2.40 & 1.50 & 2.20 \\
\hline 2 Bondgirl & 0.00 & 0.60 & 0.00 & 0.20 & 0.00 & 0.00 & 0.00 & 0.00 \\
\hline $3 \mathrm{Sam}$ & 0.00 & 0.00 & 0.00 & 0.00 & 0.00 & 0.00 & 0.00 & 0.00 \\
\hline 4 Cage & 4.00 & 3.33 & 5.00 & 5.83 & 2.50 & 1.67 & 1.50 & 0.83 \\
\hline 5 Spiky & 2.50 & 2.03 & 6.00 & 3.87 & 2.00 & 0.83 & 0.00 & 0.33 \\
\hline 6 Bella & 0.00 & 0.40 & 0.00 & 0.00 & 0.00 & 0.00 & 0.00 & 0.00 \\
\hline 7 Nikkki04 & 0.00 & 0.20 & 0.00 & 0.00 & 0.00 & 0.00 & 0.00 & 0.00 \\
\hline 8 Orangina & 2.50 & 0.50 & 0.50 & 0.17 & 0.00 & 0.00 & 0.50 & 0.00 \\
\hline 9 Arriel & 7.00 & 6.80 & 7.00 & 6.80 & 0.00 & 0.00 & 0.00 & 0.00 \\
\hline 10 Green & 5.50 & 3.67 & 6.00 & 3.86 & 4.00 & 2.25 & 1.00 & 0.00 \\
\hline 11 Valerie & 5.50 & 2.50 & 4.00 & 1.50 & 3.50 & 1.75 & 3.00 & 1.50 \\
\hline 12 Dahlia & 0.00 & 0.00 & 0.00 & 0.00 & 0.00 & 0.00 & 0.00 & 0.17 \\
\hline 13 AB123YZ & 1.50 & 0.00 & 0.00 & 0.00 & 0.00 & 0.00 & 0.00 & 0.00 \\
\hline 14 APR & 4.50 & 3.50 & 4.50 & 3.17 & 4.50 & 3.83 & 1.00 & 1.00 \\
\hline 15 Daisy & 0.00 & 0.00 & 0.50 & 0.00 & 0.00 & 0.00 & 0.50 & 0.00 \\
\hline 16 Rhonda & 5.50 & 4.17 & 3.50 & 0.50 & 3.50 & 0.33 & 2.50 & 0.00 \\
\hline 17 Lily & 0.00 & 0.00 & 0.00 & 0.00 & 0.00 & 0.00 & 0.00 & 0.00 \\
\hline
\end{tabular}

(b) Average weekly occurrence of three nonintimate sociosexual behaviors for pheromone and placebo users for the 2 baseline and 6 experimental weeks

\begin{tabular}{|c|c|c|c|c|c|c|}
\hline \multirow[b]{2}{*}{ Code name } & \multicolumn{2}{|c|}{$\begin{array}{l}\text { Informal dates (average days } \\
\text { per week occurred) }\end{array}$} & \multicolumn{2}{|c|}{$\begin{array}{l}\text { Male approaches (average days } \\
\text { per week occurred) }\end{array}$} & \multicolumn{2}{|c|}{$\begin{array}{l}\text { Masturbation (average days } \\
\text { per week occurred) }\end{array}$} \\
\hline & Baseline & Experimental & Baseline & Experimental & Baseline & Experimental \\
\hline \multicolumn{7}{|l|}{ Pheromone } \\
\hline 1 Chase & 0.00 & 0.17 & 2.00 & 2.00 & 0.50 & 2.33 \\
\hline 2 Lolita & 0.50 & 1.00 & 0.50 & 0.20 & 1.00 & 1.40 \\
\hline 3 Barbie Doll & 4.00 & 2.12 & 1.50 & 1.67 & 0.50 & 0.57 \\
\hline 4 Narcisse & 0.00 & 0.17 & 4.00 & 2.83 & 1.65 & 3.00 \\
\hline 5 Coho & 1.00 & 1.88 & 0.00 & 0.50 & 1.50 & 2.20 \\
\hline 6 Ares & 2.00 & 1.00 & 1.50 & 2.17 & 0.00 & 0.00 \\
\hline 7 Gigi & 1.50 & 0.50 & 4.00 & 4.28 & 0.00 & 0.00 \\
\hline 8 Sarah Lee & 0.00 & 0.67 & 1.50 & 2.33 & 0.00 & 0.00 \\
\hline 941799 & 1.00 & 2.40 & 2.00 & 1.50 & 0.00 & 0.00 \\
\hline 10 Lynette & 1.50 & 1.33 & 5.00 & 3.67 & 0.00 & 0.00 \\
\hline 11 Potus & 3.00 & 3.00 & 4.00 & 3.00 & 0.00 & 0.00 \\
\hline 12 Randall & 2.50 & 0.40 & 3.50 & 1.80 & 2.00 & 1.60 \\
\hline 13 Saffron & 0.00 & 0.00 & 0.50 & 0.40 & 1.50 & 1.17 \\
\hline
\end{tabular}


Table 4 (continued)

(b) Average weekly occurrence of three nonintimate sociosexual behaviors for pheromone and placebo users for the 2 baseline and 6 experimental weeks

\begin{tabular}{|c|c|c|c|c|c|c|}
\hline \multirow[b]{2}{*}{ Code name } & \multicolumn{2}{|c|}{$\begin{array}{l}\text { Informal dates (average days } \\
\text { per week occurred) }\end{array}$} & \multicolumn{2}{|c|}{$\begin{array}{l}\text { Male approaches (average days } \\
\text { per week occurred) }\end{array}$} & \multicolumn{2}{|c|}{$\begin{array}{l}\text { Masturbation (average days } \\
\text { per week occurred) }\end{array}$} \\
\hline & Baseline & Experimental & Baseline & Experimental & Baseline & Experimental \\
\hline \multicolumn{7}{|l|}{ Pheromone } \\
\hline 14 Absinthe & 1.50 & 2.24 & 5.00 & 4.44 & 3.50 & 2.28 \\
\hline 15 Psychokity & 0.50 & 0.33 & 2.50 & 1.33 & 0.50 & 0.33 \\
\hline 16 Sammi & 0.00 & 2.20 & 2.00 & 3.00 & 1.00 & 0.80 \\
\hline 17 Yvette & 3.00 & 2.17 & 0.50 & 1.00 & 1.50 & 0.50 \\
\hline 18 Jeneve & 0.00 & 1.20 & 3.50 & 3.20 & 1.00 & 0.80 \\
\hline 19 Sunset & 1.50 & 1.00 & 3.00 & 1.40 & 1.00 & 0.80 \\
\hline \multicolumn{7}{|l|}{ Placebo } \\
\hline 1 Red & 0.50 & 3.00 & 3.00 & 4.40 & 0.00 & 0.00 \\
\hline 2 Bondgirl & 0.00 & 0.60 & 0.00 & 1.00 & 0.00 & 0.00 \\
\hline $3 \mathrm{Sam}$ & 0.00 & 0.17 & 0.00 & 0.33 & 0.00 & 0.00 \\
\hline 4 Cage & 0.00 & 0.50 & 0.50 & 0.50 & 2.00 & 1.67 \\
\hline 5 Spiky & 0.00 & 0.23 & 1.00 & 1.57 & 2.00 & 2.17 \\
\hline 6 Bella & 0.00 & 0.40 & 1.50 & 2.60 & 0.00 & 0.00 \\
\hline 7 Nikkki04 & 0.00 & 0.20 & 1.50 & 1.20 & 0.00 & 0.40 \\
\hline 8 Orangina & 0.50 & 1.00 & 4.00 & 3.67 & 0.00 & 0.00 \\
\hline 9 Arriel & 0.00 & 0.00 & 0.50 & 0.00 & 1.50 & 0.40 \\
\hline 10 Green & 2.50 & 1.61 & 0.50 & 0.33 & 1.50 & 0.17 \\
\hline 11 Valerie & 3.00 & 2.25 & 0.50 & 0.50 & 0.00 & 1.25 \\
\hline 12 Dahlia & 0.00 & 0.00 & 1.00 & 0.67 & 0.00 & 0.00 \\
\hline $13 \mathrm{AB} 123 \mathrm{YZ}$ & 0.00 & 0.00 & 1.50 & 1.83 & 0.00 & 0.50 \\
\hline 14 APR & 5.00 & 2.67 & 1.50 & 1.83 & 1.00 & 0.33 \\
\hline 15 Daisy & 1.00 & 0.67 & 1.50 & 1.00 & 1.00 & 0.33 \\
\hline 16 Rhonda & 1.00 & 0.33 & 2.00 & 5.00 & 3.00 & 1.50 \\
\hline 17 Lily & 1.00 & 0.17 & 3.50 & 2.67 & 0.00 & 0.00 \\
\hline
\end{tabular}

excluded from tabulation. By starting on Day 8, the protocol avoided bleeding days yet still allowed for 2 baseline and 6 experimental weeks of data for the majority of subjects. Table 2 shows the definitions of the 2 baseline and 6 experimental weeks.

\subsection{Perceptions}

Women were asked to record whether they had noticed any change in their "experiences with men" each week. The number of individuals perceiving positive changes during any of the experimental weeks was counted.

\subsection{Data analysis}

Differences between groups were tested for age, height, weight, body mass index and menstrual cycle length at baseline and during the first experimental cycle using $t$ tests. We also examined group differences for dating status at the beginning and end of the study, ethnicity, reported accuracy and back-filling of the data, perception of a positive effect and rate of perfume use.

For sociosexual behavior, a subject was characterized as showing an increase over baseline when her average weekly score for the 6-week experimental period exceeded her average weekly score for the 2-week baseline period. When menstrual cycle length was less than 28 days, interpolation of average days of behavior for the third week was calculated as long as data for at least 5 of 7 days were available. When 5 days were not available due to onset of menses, the partial week of data was not used. The interpolation formula used was as follows: if 6 of 7 days of data were available, the actual behavioral count was multiplied by $7 / 6$ to yield the interpolated weekly count. Thus, if a subject recorded 4 days of behavior over a 6-day period $(7 / 6 \times 4)$, the interpolated frequency is 4.66 . Because of the considerable variability in the 36 subjects' behavior at baseline, the key question of interest was whether a subject increased her average weekly behavior during the experimental period over her own average weekly behavior at baseline. For each behavior, a $\chi^{2}$ probability test was used to test for differences between the two groups in the proportion of subjects showing an increase over baseline in average weekly behavior.

\subsection{Statistical comments}

Regardless of where a woman started at baseline, the question this experiment was designed to test was "Did a woman increase in average weekly behavior over her own average weekly baseline when she added this pheromone to her perfume and were there significantly more such positive effects for pheromone subjects than for those who used placebo?" 
Table 5

Effects of pheromones vs. placebo on increase over baseline for each of the seven sociosexual behaviors

\begin{tabular}{|c|c|c|c|c|c|c|c|}
\hline Code name & Petting/kissing/affection & $\begin{array}{l}\text { Sleeping next to a } \\
\text { romantic partner }\end{array}$ & $\begin{array}{l}\text { Sexual } \\
\text { intercourse }\end{array}$ & $\begin{array}{l}\text { Formal } \\
\text { dates }\end{array}$ & $\begin{array}{l}\text { Informal } \\
\text { dates }\end{array}$ & $\begin{array}{l}\text { Male } \\
\text { approaches }\end{array}$ & Masturbation \\
\hline \multicolumn{8}{|l|}{ Pheromone } \\
\hline 1 Chase & $\Uparrow$ & $\Uparrow$ & $\Uparrow$ & $\Uparrow$ & $\Uparrow$ & & $\Uparrow$ \\
\hline 2 Gigi & $\Uparrow$ & $\Uparrow$ & $\Uparrow$ & $\Uparrow$ & & $\Uparrow$ & \\
\hline 3 Lolita & $\Uparrow$ & $\Uparrow$ & $\Uparrow$ & & $\Uparrow$ & & $\Uparrow$ \\
\hline 4 Barbie Doll & & $\Uparrow$ & $\Uparrow$ & $\Uparrow$ & & $\Uparrow$ & $\Uparrow$ \\
\hline 5 Randall & $\Uparrow$ & $\Uparrow$ & $\Uparrow$ & $\Uparrow$ & & & \\
\hline 6 Saffron & $\Uparrow$ & $\Uparrow$ & $\Uparrow$ & $\Uparrow$ & & & \\
\hline 7 Sarah Lee & $\Uparrow$ & & & $\Uparrow$ & $\Uparrow$ & $\Uparrow$ & \\
\hline 8 Narcisse & $\Uparrow$ & & $\Uparrow$ & & $\Uparrow$ & & $\Uparrow$ \\
\hline 9 Coho & & $\Uparrow$ & & & $\Uparrow$ & $\Uparrow$ & $\Uparrow$ \\
\hline 1041799 & $\Uparrow$ & $\Uparrow$ & & & $\Uparrow$ & & \\
\hline 11 Psychokity & $\Uparrow$ & & $\Uparrow$ & $\Uparrow$ & & & \\
\hline $12 \mathrm{Sammi}$ & $\Uparrow$ & & & & $\Uparrow$ & $\Uparrow$ & \\
\hline 13 Absinthe & & $\Uparrow$ & & $\Uparrow$ & $\Uparrow$ & & \\
\hline 14 Yvette & & $\Uparrow$ & $\Uparrow$ & & & $\Uparrow$ & \\
\hline 15 Jeneve & $\Uparrow$ & & & & $\Uparrow$ & & \\
\hline 16 Lynette & & $\Uparrow$ & & $\Uparrow$ & & & \\
\hline 17 Ares & & & & $\Uparrow$ & & $\Uparrow$ & \\
\hline \multicolumn{8}{|l|}{18 Potus } \\
\hline \multicolumn{8}{|l|}{19 Sunset } \\
\hline Increased over baseline $(n)$ & 11 & 11 & 9 & 10 & 9 & 7 & 5 \\
\hline Proportion of group (\%) & 57.9 & 57.9 & 47.4 & 52.6 & 47.4 & 36.8 & 26.3 \\
\hline \multicolumn{8}{|l|}{ Placebo } \\
\hline 1 Red & $\Uparrow$ & $\Uparrow$ & $\Uparrow$ & $\Uparrow$ & $\Uparrow$ & $\Uparrow$ & \\
\hline 2 Bondgirl & $\Uparrow$ & $\Uparrow$ & & & $\Uparrow$ & $\Uparrow$ & \\
\hline 3 Spiky & & & & $\Uparrow$ & $\Uparrow$ & $\Uparrow$ & $\Uparrow$ \\
\hline 4 Bella & $\Uparrow$ & & & & $\Uparrow$ & $\Uparrow$ & \\
\hline 5 Nikkki04 & $\Uparrow$ & & & & $\Uparrow$ & & $\Uparrow$ \\
\hline $6 \mathrm{Sam}$ & & & & & $\Uparrow$ & $\Uparrow$ & \\
\hline 7 Cage & & $\Uparrow$ & & & $\Uparrow$ & & \\
\hline $8 \mathrm{AB} 123 \mathrm{YZ}$ & & & & & & $\Uparrow$ & $\Uparrow$ \\
\hline 9 APR & & & & & & $\Uparrow$ & \\
\hline 10 Rhonda & & & & & & $\Uparrow$ & \\
\hline 11 Orangina & & & & & $\Uparrow$ & & \\
\hline 12 Dahlia & & & & $\Uparrow$ & & & \\
\hline 13 Valerie & & & & & & & $\Uparrow$ \\
\hline \multicolumn{8}{|l|}{14 Arriel } \\
\hline \multicolumn{8}{|l|}{15 Green } \\
\hline \multicolumn{8}{|l|}{16 Daisy } \\
\hline \multicolumn{8}{|l|}{17 Lily } \\
\hline Increased over baseline $(n)$ & 4 & 3 & 1 & 3 & 8 & 8 & 4 \\
\hline Proportion of group (\%) & 23.5 & 17.6 & 5.9 & 17.6 & 47.1 & 47.1 & 23.5 \\
\hline
\end{tabular}

The average weekly frequency of sexual intercourse of a group of pheromone users is an irrelevant measure in a protocol testing an individual's increase in average weekly behavior over her own baseline. What matters in the life of a subject is whether she increased over her own baseline. By establishing an experiment to test the proportion of individuals who increased over baseline, we avoided the error in logic that the ANOVA- or Mann-Whitney-type statistics would impose. Other than a baseline comparison between groups to rule out the probably irrelevant possibility of a significant difference in behavior, the absolute amount of behavior cannot be compared between groups in hopes of offering meaningful information nor should individuals be compared to each other and ranked in either their absolute amount of average weekly behavior or absolute numerical change in average weekly behavior. The proportion of pheromone users who increased over baseline is the correct variable for comparison with the placebo group.

\section{Results}

\subsection{Perfume use, perceptions, cycle lengths and dating status}

The 36 women in this experiment chose 34 different fragrances rendering the potential impact of a particular fragrance moot. During the 6 experimental weeks, daily calendars showed an average use of the test perfume of 5.4 
Table 6

Number of subjects with an increase over baseline for each of 7 sociosexual behaviors by treatment group

\begin{tabular}{|c|c|c|c|c|c|}
\hline \multirow[b]{3}{*}{ Sociosexual behavior } & \multicolumn{5}{|c|}{ Treatment } \\
\hline & \multicolumn{2}{|c|}{$\begin{array}{l}\text { Pheromone } \\
(n=19)\end{array}$} & \multicolumn{2}{|c|}{$\begin{array}{l}\text { Placebo } \\
(n=17)\end{array}$} & \multirow[b]{2}{*}{$P$} \\
\hline & $n$ & $\%$ & $n$ & $\%$ & \\
\hline Petting/affection/kissing & 11 & 57.9 & 4 & 23.5 & $<.04$ \\
\hline Sleeping next to a romantic partner & 11 & 57.9 & 3 & 17.6 & $<.02$ \\
\hline Sexual intercourse & 9 & 47.4 & 1 & 5.9 & $<.006$ \\
\hline Formal dates & 10 & 52.6 & 3 & 17.6 & $<.03$ \\
\hline Informal dates & 9 & 47.4 & 8 & 47.1 & ns \\
\hline Male approaches & 7 & 36.8 & 8 & 47.1 & ns \\
\hline Masturbation & 5 & 26.3 & 4 & 23.5 & ns \\
\hline
\end{tabular}

$($ S.D. $=1.3)$ times per week for pheromone users and 5.3 $($ S.D. $=1.3)$ times per week for placebo users. Usage did not differ significantly between the two groups $[t(35)<1]$.

During this experimental period, 9 (47\%) pheromone users compared with $7(41.2 \%)$ placebo users reported the perception of positive results in their experiences with men, and this difference was not significant $\left[\chi^{2}(1, n=36) \leq 1\right]$. On the debriefing form, $88.9 \%$ of the pheromone and $88.2 \%$ of the placebo group scored themselves as mostly or very accurate $\left[\chi^{2}(1, n=35) \leq 1\right]$ and only $10.1 \%$ and $17.7 \%$ of women, respectively, reported back-filling their data 'about $\{1 / 2\}$ ' the time or more, and this difference was not significant $\left[\chi^{2}(1, n=35) \leq 1\right]$.

The average menstrual cycle length during the first experimental cycle for pheromone subjects was 28.9 days (S.D. $=7.9)$ compared with 27.6 days (S.D. $=4.6)$ for placebo subjects. The difference was not significantly different $[t(34)<1]$. Comparing the length of each subject's baseline menstrual cycle with her first experimental cycle revealed no significant change for either group. For 19 pheromone users, average menstrual cycle length difference between the baseline cycle and the first experimental cycle was -0.11 days $[t(18)<1]$. For 17 placebo users, this same difference was 1.88 days $[t(16)=2.24$ (S.E. $=1.37)]$ and was not significant. The average change in menstrual cycle length for the placebo group compared with the pheromone group was not significant $[M=1.99, t(34)<1]$.

Women reported their dating status as not dating, dating or dating steadily. No appreciable difference occurred between groups at baseline, at end or as a change in dating status. Baseline records of dating status of the 19 pheromone users revealed 8 not dating and 11 dating. For 17 placebo users, 9 were not dating and 8 were dating $\left[\chi^{2}(1\right.$, $n=36) \leq 1$ ]. Dating status reported for the last week of the study for the 19 pheromone users revealed 6 not dating and 13 dating. For 17 placebo users, 7 were not dating and 10 were dating $\left[\chi^{2}(1, n=36) \leq 1\right]$. Increases in dating status occurred in seven pheromone and five placebo users, decreases in dating status occurred in four pheromone and three placebo users and there was no change for eight pheromone and nine placebo users $\left[\chi^{2}(2, n=36) \leq 1\right]$.

\subsection{Sociosexual behavior}

The major hypothesis was that a greater proportion of pheromone $(\mathrm{Ph})$ than placebo $(\mathrm{Pl})$ users would show an increase over baseline. With the exception of male approaches $[\mathrm{Ph}=2.4, \mathrm{Pl}=1.4 ; t(34)=2.24, P<.04]$, average weekly frequencies of sociosexual behaviors for placebo and pheromone groups were not significantly different at baseline (see Table 3).

The average number of days per week of behaviors recorded by each subject during the baseline and experimental phase of the investigation are presented in Table $4 \mathrm{a}$

Table 7

Cumulative number of sociosexual behaviors involving a partner whose weekly averages increased above baseline for pheromone and placebo users

\begin{tabular}{|c|c|c|c|c|c|c|}
\hline \multirow[b]{2}{*}{ Code name } & \multicolumn{6}{|c|}{$\begin{array}{l}\text { Number of behaviors that increase } \\
\text { above baseline }\end{array}$} \\
\hline & $\geq 1$ & $\geq 2$ & $\geq 3$ & $\geq 4$ & $\geq 5$ & $\geq 6$ \\
\hline \multicolumn{7}{|l|}{ Pheromone } \\
\hline 1 Chase & $\times$ & $\times$ & $\times$ & $\times$ & $\times$ & \\
\hline 2 Gigi & $\times$ & $\times$ & $\times$ & $\times$ & $\times$ & \\
\hline 3 Lolita & $\times$ & $x$ & $x$ & $x$ & & \\
\hline 4 Barbie Doll & $\times$ & $\times$ & $\times$ & $\times$ & & \\
\hline 5 Randall & $\times$ & $x$ & $x$ & $\times$ & & \\
\hline 6 Saffron & $\times$ & $\times$ & $\times$ & $\times$ & & \\
\hline 7 Sarah Lee & $\times$ & $x$ & $x$ & $\times$ & & \\
\hline 8 Narcisse & $\times$ & $x$ & $\times$ & & & \\
\hline 9 Coho & $x$ & $x$ & $x$ & & & \\
\hline 1041799 & $x$ & $x$ & $x$ & & & \\
\hline 11 Psychokity & $\times$ & $\times$ & $\times$ & & & \\
\hline 12 Sammi & $\times$ & $x$ & $\times$ & & & \\
\hline 13 Absinthe & $\times$ & $\times$ & $\times$ & & & \\
\hline 14 Yvette & $\times$ & $x$ & $x$ & & & \\
\hline 15 Jeneve & $x$ & $\times$ & & & & \\
\hline 16 Lynette & $\times$ & $x$ & & & & \\
\hline 17 Ares & $\times$ & $x$ & & & & \\
\hline \multicolumn{7}{|l|}{18 Potus } \\
\hline \multicolumn{7}{|l|}{19 Sunset } \\
\hline Increased over baseline $(n)$ & 17 & 17 & 14 & 7 & 2 & 0 \\
\hline Proportion of group (\%) & 89.5 & 89.5 & 73.7 & 36.9 & 10.5 & 0 \\
\hline \multicolumn{7}{|l|}{ Placebo } \\
\hline 1 Red & $\times$ & $\times$ & $\times$ & $x$ & $\times$ & $\times$ \\
\hline 2 Bondgirl & $\times$ & $x$ & $x$ & $x$ & & \\
\hline 3 Spiky & $\times$ & $x$ & $\times$ & & & \\
\hline 4 Bella & $\times$ & $x$ & $\times$ & & & \\
\hline 5 Nikkki04 & $x$ & $x$ & & & & \\
\hline $6 \mathrm{Sam}$ & $\times$ & $x$ & & & & \\
\hline 7 Cage & $\times$ & $x$ & & & & \\
\hline $8 \mathrm{AB} 123 \mathrm{YZ}$ & $\times$ & & & & & \\
\hline 9 APR & $\times$ & & & & & \\
\hline 10 Rhonda & $\times$ & & & & & \\
\hline 11 Orangina & $\times$ & & & & & \\
\hline 12 Dahlia & $\times$ & & & & & \\
\hline \multicolumn{7}{|l|}{13 Valerie } \\
\hline \multicolumn{7}{|l|}{14 Arriel } \\
\hline \multicolumn{7}{|l|}{15 Green } \\
\hline \multicolumn{7}{|l|}{16 Daisy } \\
\hline \multicolumn{7}{|c|}{17 Lily } \\
\hline Increased over baseline $(n)$ & 12 & 7 & 4 & 2 & 1 & 1 \\
\hline Proportion of group (\%) & 70.6 & 41.2 & 23.5 & 11.8 & 5.9 & 5.9 \\
\hline
\end{tabular}

Global effects: $\chi^{2}=12.54, d f=1, P<.001$. 
and $b$. Table $4 a$ arrays the data of the four most intimate sociosexual behaviors. Table $4 \mathrm{~b}$ arrays the data of the three least intimate sociosexual behaviors.

The effects of the pheromone and placebo formulas on increase over baseline for each of the seven sociosexual behaviors are shown in Table 5 .

When compared with placebo users, a significantly higher proportion of pheromone users showed an increase over baseline for frequency of sexual intercourse $\left[\chi^{2}(1\right.$, $n=36)=7.70]$, sleeping next to a romantic partner $\left[\chi^{2}(1\right.$, $n=36)=6.12]$, formal dates $\left[\chi^{2}(1, n=36)=4.76\right]$ and petting/affection/kissing $\left[\chi^{2}(1, n=36)=4.36\right]$. No significant effects of pheromones were observed in the proportion of subjects who showed an increase over baseline in frequency of informal dates, male approaches or masturbation (see Table 6).

The cumulative number of increases in sociosexual behaviors involving a partner that occurred during the experimental phase is displayed in Table 7. Both visual inspection of the density and statistical evaluation of the global effects reveal a significantly and substantially greater effect of pheromones.

The protocol developed in advance for this study was designed to measure behavior during two menstrual cycles in order to allow a 6-week experimental period similar to the experiment with sex attractant pheromones in men [7]. However, Stern and McClintock [17] reported shifting the timing of ovulation within one cycle with their extracts of underarm secretions. We therefore did a post hoc test of the effects examining data from the first experimental cycle only. Comparisons of the placebo with the pheromone group revealed statistically significant positive effects for sexual intercourse $\left[\chi^{2}(1, n=36)=4.98\right.$, $P<.03]$ and formal dating $\left[\chi^{2}(1, n=36)=4.12, P<.04\right]$, with kissing/petting/affection achieving borderline significance $\left[\chi^{2}(1, n=36)=2.95, P=.08\right]$.

\section{Discussion}

In this prospective double-blind, placebo-controlled study, we investigated the sex attractant effects of a putative synthesized pheromone formulated on the basis of prior research. The topical application of this putative female sex attractant pheromone caused a statistically significant and distinct increase over average weekly baseline in four sociosexual behaviors: sexual intercourse, sleeping next to a romantic partner, formal dates and petting/affection/kissing. There was no significant increase in male approaches, masturbation or informal dates.

It is appropriate to question the nature of the makeup of the placebo and treatment groups as they differed on several key variables that conceivably could have influenced the outcome of the study. The diverse ethnic array reflected the composition of the psychology classes at San Francisco State University where the call for subjects had been announced. There are currently no data in the literature that would suggest differences among ethnic groups in pheromone susceptibility or excretion. However, the pheromone treatment group tended to be younger $(P=.09)$ and shorter $(P<.05)$ and reported being approached by men more often $(P<.05)$ than the placebo group at baseline. Balancing these factors, we note that the placebo group tended to sleep next to a romantic partner more often $(P<.10)$, and although they tended to be taller, their body mass index was not different from the pheromone group. Given the shorter stature and the younger age of the pheromone group, these variables might have affected the outcome and were therefore examined further. Two post hoc questions were tested comparing women at baseline using $t$ tests with a median split: (1) Of the 35 women who provided data on height, were the shorter women in the total group comparable to those who were taller in the six sociosexual behaviors? and (2) Of the entire group of 36 women who provided date of birth, were the younger women comparable to the older women? Results showed that shorter height and younger age were not associated with any significant differences in average weekly frequency of baseline sexual intercourse, sleeping next to a romantic partner, petting/affection/kissing, formal dates, informal dates or male approaches.

Confidence in our findings is strengthened by the fact that the self-reported percentage of the time subjects back-filled was low, the perceived accuracy of self-report was high and there were no significant differences between the two groups for either back-filling or accuracy. Perception of positive effects of perfume use in the two groups was moderate and not significantly different. There was no indication that subjects could tell which product they had been using. Thus, we reject the possibility that changes in behavior were due simply to expectations. While we look forward to learning what the chemical composition of Athena Pheromone 1013 is when the patent process has been completed, that missing information about chemistry and potential mechanism of action does not impede our understanding of the behavioral effects of the formula.

It is particularly noteworthy that the proportion of our subjects recording an increase over baseline in masturbation was low (less than 27\%) and the two groups were not significantly different. If masturbation is seen as an indicator of sexual motivation, then the synthesized pheromone formula did not affect the sexual motivation of our subjects. By the widely accepted definition of the term, a pheromone is expected to cause a change in reproductive behavior of another, usually of the same species. The results of this study suggest that the synthesized female pheromones used here had positive sexual attractant effects that were fundamentally the same as those that had been found previously with synthesized male sex attractant pheromones used by men [7].

Three prior experiments [15-17] have applied extracts of underarm sweat vs. placebo and shown that the timing of the 
menstrual cycle was altered. Our experiment did not show an alteration in menstrual cycle, implying that this test pheromone has a different composition than that of whole extracts of underarm sweat.

Robustness of these effects was evident. Three or more behaviors increased over baseline in $74 \%$ of the women using pheromones, an amount substantially and significantly higher than the $23 \%$ of placebo subjects showing such an increase. The effect of the pheromone formula was comparable to that reported in two recent studies investigating pheromone effects in double-blind research in humans $[7,17]$. For men, the increase over baseline for three or more sociosexual behaviors occurred in $41 \%$ of pheromone and $9.5 \%$ of placebo users [7]. For women, $68 \%$ of subjects using extracts of female underarm secretions experienced positive effects in shifting the timing of ovulation, a different effect of pheromones [17]. The pheromone formula used in our study had no effect on menstrual cycle length.

We deduce that sexual attractiveness is what increased in the pheromone users. Significantly more women using pheromones increased in their frequency over baseline in four intimate sociosexual behaviors with men. Because male approaches at baseline was significantly higher for the pheromone group, our failure to find a significantly higher proportion of pheromone users who increased over baseline in male approaches may have been related to this difference. As was true for the men in the study of male pheromones [7], the biggest difference between the placebo and pheromone groups in our study was for the most reproductively important behaviors: sexual intercourse and sleeping next to a romantic partner.

Significant effects appeared to occur within the first cycle and the proportion of women recording effects increased by the second cycle. Future studies might investigate whether those women using pheromones, who do not record an increase in these sociosexual behaviors in the first or the second cycle, might still increase if the duration of the study were extended.

We conclude that this synthesized female pheromone formula, applied topically, acted as a sex attractant pheromone and increased the sexual attractiveness of women to men. The results of this study strongly suggest that substances occurring naturally in the underarm secretions of young, fertile, sexually active women have powerful effects on the behavior of the other sex. A woman who exudes such substances is apparently a more sexually attractive indi- vidual, and as a consequence, men's sociosexual interaction with such women is significantly increased.

\section{References}

[1] Benton B. The influence of androstenol-a putative human pheromone — on mood throughout the menstrual cycle. Biol Psychol 1982; 15:249-56.

[2] Brennan PA, Keverne EB. Neural mechanisms of mammalian olfactory learning. Prog Neurobiol 1997;51:457-81.

[3] Cutler WB. Pheromonal modulation of brain and behavior. In: Halbreich $\mathrm{U}$, editor. Hormonal modulation of brain and behavior. Washington, DC: American Psychiatric Press, 2002, in press.

[4] Cutler WB. Human sex-attractant pheromones: discovery, research, development, and application in sex therapy. Psychiatr Ann 1999; 29:54-9.

[5] Cutler WB. Female essence (pheromones) increases sexual behavior of young women (abstract). Neuroendocrinol Lett 1987;9(3):199.

[6] Cutler WB, Stine R. Female essence increases heterosexual activity of women. Abstracts: scientific papers to be presented at the forty-fourth annual meeting of the American Fertility Society. Birmingham, AL: American Fertility Society, 1988. p. S110.

[7] Cutler WB, Friedmann E, McCoy NL. Pheromonal influences on sociosexual behavior of men. Arch Sex Behav 1998;27:1-13.

[8] Cutler WB, Preti G, Krieger A, Huggins GR, Garcia CR, Lawley HJ. Human axillary secretions influence women's menstrual cycles: the role of donor extract from men. Horm Behav 1986;20:463-73.

[9] Eysenck HJ, Eysenck SBG. Eysenck personality questionnaire-revised. San Diego, CA: Educational and Industrial Testing Service, 1993.

[10] Jacob S, McClintock MK. Psychological state and mood effects of steroidal chemosignals in women and men. Horm Behav 2000;37: $57-78$.

[11] Johnson W, Zava D, McCoy N. Overall self-confidence, self-confidence in mathematics, and sex-role stereotyping in relation to salivary free testosterone in university women. Percept Motor Skills 2000; 91:391-401

[12] Karlson P, Luscher M. Pheromones: a new term for a class of biologically active substances. Nature 1959;183:55-6.

[13] Lundstrom NJ, Olsson MJ, Larsson M. Effects of the putative pheromone 4,16-androstadien-3-one on psychological and psychophysiological variables: weak evidence. Chem Sens 2000;25:613.

[14] Monti-Bloch L, Diaz-Sanchez V, Jennings-White C, Berliner DL. Modulation of serum testosterone and autonomic function through stimulation of the male human vomeronasal organ (VNO) with pregna-4,20-diene-3,6-dione. J Steroid Biochem Mol Biol 1998; 65:237-42.

[15] Preti G, Cutler WB, Garcia CR, Krieger A, Huggins GR, Lawley HJ. Human axillary secretions influence women's menstrual cycles: the role of donor extract of females. Horm Behav 1986;20:474-82.

[16] Preti G, Cutler WB, Christensen CM, Lawley H, Huggins GR, Garcia CR. Human axillary extracts: analysis of compounds from samples which influence menstrual timing. J Chem Ecol 1987; 13:717-31.

[17] Stern K, McClintock MK. Regulation of ovulation by human pheromones (Letter). Nature 1998;392:177-9. 